\title{
Impact of MRSA on the Military Medical Service and Diagnostic Point-of-Care Options for the Field Setting
}

\author{
Hagen Frickmann ${ }^{1,2 *}$ \\ ${ }^{1}$ Department of Microbiology and Hospital Hygiene, Bundeswehr Hospital Hamburg, \\ Bernhard Nocht Str. 74, 20359 Hamburg, Germany \\ ${ }^{2}$ Department of Medical Microbiology, Virology and Hygiene, University Medicine Rostock, \\ Schillingallee 70, 18057 Rostock, Germany
}

Received: 25 May 2018; accepted: 01 June 2018

\begin{abstract}
Methicillin-resistant Staphylococcus aureus (MRSA) poses an infection risk for international military deployments. In the presented mini-review, the history of MRSA in the medical service and modern warfare is highlighted. To allow rapid diagnosis, various molecular diagnostic point-of-care solutions are available. Most evaluation studies, however, are focused on screening swabs rather than clinical materials and evaluation data from harsh environments are widely lacking. Accordingly, studies with complex sample materials under difficult environmental conditions, e.g., in the desert or in the tropics, are desirable to close this gap of knowledge regarding the diagnostic reliability of such modern molecular point-of-care devices.
\end{abstract}

Keywords: MRSA, molecular detection, molecular point of care testing, military medicine, transmission prevention

\section{Impact of Methicillin-Resistant Staphylococcus aureus on the Military Medical Service}

Methicillin-resistant Staphylococcus aureus (MRSA) has been a menace to the military medical service for decades. As early as in the $1980 \mathrm{~s}$, an outbreak of MRSA was described in a British Royal Navy hospital [1]. After this, multiple publications on this issue followed, including reports from deployment sites. For example, during an assessment of 2242 US casualties from Operation Iraqi Freedom and Operation Enduring Freedom in the first decade of the present century, MRSA was among the three most frequently isolated multidrug-resistant pathogens associated with nosocomial infection rates less than 5\% [2].

Nosocomial transmission of MRSA in military settings is of particular relevance in very constricted environments, e.g., on board of seagoing military vessels. Onboard of US American warships, prevalence of 3.5\% (17/400) MRSA colonization was observed. No specific risk factors were identified, suggesting that the environment itself might be a problem. Also, $198(49.5 \%)$ soldiers were colonized with methicillin-sensitive S. aureus (MSSA) [3].

Similarly, limited living conditions exist in military barracks. In case of staff skin lesions in military barracks, however, the differential diagnosis of MRSA infections is often neglected and alternative hypotheses like spider bites seem more plausible to soldiers [4].

Especially, strains which are positive for Panton-Valentine leukocidin (PVL) showed a clear tendency of progression from colonization of the skin to soft tissue infections in US soldiers [5]. Thereby, PVL is an epidemiological marker for strains with pronounced invasiveness which are associated with severe wound infections. Nevertheless, PVL is not the exclusive cause of increased pathogenic potential. Instead, various factors including phenol-soluble modulins (PSM) have an

\footnotetext{
* Corresponding author: Department of Microbiology and Hospital Hygiene Bundeswehr Hospital Hamburg, Bernhard Nocht street 74, D-20359 Hamburg, Germany; E-mail: Frickmann@bnitm.de, hagen.frickmann@med.uni-rostock.de.
}

equal or even bigger role in this process [6-10]. Anyway, wound infections are highly relevant in military deployment settings, resulting in a variety of studies in this field.

Thereby, MRSA infections are rarely observed in early postsurgical wound infections. In particular, only 2 out of 49 cases of very early wound stages in casualties in Iraq were associated with MRSA detection [11]. Such results make nosocomial transmission highly likely.

MRSA prevalence is regularly monitored by the US armed forces also in their home country. In the USA, communityacquired MRSA is infrequently detected at military training units. The frequency ranges between 27 and 32 MRSA infections per 1000 soldiers [12]. Mupirocin-based eradication is effective but neither prevents recolonization nor does it reduce the infection rate in soldiers [13]. Next to military training camps, community-acquired MRSA strains were also infrequently (9 out of 67 [13.4\%] total MRSA cases) observed in patients without identified risk factors in a US military hospital [14]. Generally, community-acquired colonization with MRSA in US soldiers was shown to be associated with previous antibiotic therapy [5].

The high relevance of MRSA for military deployments makes rapid diagnostic detection an issue of importance. Rapid and easy-to-apply molecular diagnostic options are therefore detailed in the following.

\section{Diagnostic Point-of-Care Solutions for Potential Use on Deployment}

Rapid MRSA detection is in the focus of molecular RDT (rapid diagnostic test) approaches. Rapid identification of MRSA using the Xpert MRSA/SA (Cepheid) RDT system was recently shown to contribute to optimized antimicrobioal management in a small proof-of-principle study with positive blood cultures in obstetric patients [15]. In a recent evaluation from Denmark with screening swabs and a culture-based gold standard including broth enrichment, sensitivity, specificity, positive and negative predictive

This is an open-access article distributed under the terms of the Creative Commons Attribution-NonCommercial 4.0 International License (https://creativecommons.org/licenses/by-nc/4.0/), which permits unrestricted use, distribution, and reproduction in any medium for non-commercial purposes, provided the original author and source are credited, a link to the CC License is provided, and changes - if any - are indicated. 
value of the Xpert MRSA Gen 3 system were $88.2 \%$, 97.9\%, $62.5 \%$, and $99.5 \%$, respectively, with hands-on time of $8.8 \mathrm{~min}$ and mean laboratory turnaround time of 2.9 (1-6) hours [16]. Similarly good results for the Xpert MRSA assay were shown by an Irish study with sensitivity, specificity, and positive and negative predictive values of $95 \%, 98 \%, 90 \%$, and $99 \%$, respectively, for nasal swabs and $90 \%, 97 \%, 86 \%$, and $98 \%$, respectively, for swabs from nose, throat, and groin/perineum sites. Throat swabs scored worst with $75 \%$ sensitivity. The limit of detection (LOD) was estimated to be 610 cfu (colony forming units) $/ \mathrm{mL}$ or 58 cfu per swab [17].

When applying MRSA PCR on swabs, however, one has to bear in mind that there is the risk of deodorant/anti-perspirantinduced invalidation of axillary PCR samples as observed in an evaluation of the Xpert SA Nasal Complete PCR by the US military [18]. In addition, a French study group reported sensitivity problems of the Xpert MRSA/SA Nasal system in association with a sample collection which contained phenotypic MRSA isolates with the mecA homologue mecALGA251 [19].

An evaluation of the Xpert MRSA/SA technique for the detection of coagulase-negative staphylococci in periprosthetic joint infections showed sensitivity, specificity, positive and negative predictive value of $36 \%, 98 \%, 90 \%$, and $74 \%$, respectively, so the approach had to be dropped due to poor sensitivity [20].

In a study with positive-blood-culture broths, the Xpert MRSA/SA BC system showed sensitivity of 98.1\% (range, $87.5 \%-100 \%$ ) and specificity of $99.6 \%$ (range, $98.3 \%-100 \%$ ) for the identification of MRSA [21]. Similarly good results for blood culture materials were detected by other authors [22-25].

The commercial loop-mediated amplification (LAMP)-based eazyplex MRSA assay (AmplexDiagnostics) showed sensitivity of $83.3 \%$ and specificity of $97.8 \%$ for $S$. aureus detection in pleural and synovial fluid with an LOD of $6.4 \times 10^{3} \mathrm{cfu} / \mathrm{ml}$ for S. aureus and $1.0 \times 10^{4} \mathrm{cfu} / \mathrm{mL}$ for MRSA [26]. Of note, the eazyplex system was also designed to target mecC-based resistance.

The Filmarray system (BioFire Diagnostics, Inc., Salt Lake City, UT, USA), another molecular tool for potential use in the field, includes an option for the detection of MRSA from blood cultures in its blood culture identification panel. In detail, three resistance genes (mecA, vanA/B, and bla $\left.a_{K P C}\right)$ are targeted and allow for the detection of $m e c A$-associated MRSA strains. In an eightcenter trial with 2207 positive aerobic blood culture samples, sensitivity and specificity were $98.4 \%$ and $98.3 \%$ for $m e c A$ gene detection, respectively [27]. In a South African study, consistency with the reference methods was even as good as $100 \%$ [28].

\section{Conclusions}

Various molecular point-of-care approaches for the diagnosis of MRSA are available and potentially suitable for use on deployment. Nevertheless, there is still some evaluation work to be done. Evaluation data from deployment sites with harsh environmental effects, e.g., in the desert or in the tropics, are scarcely available. Furthermore, most of the evaluations have focused on swabs, which usually detect mere colonization rather than real infections. Therefore, broader evaluations with more complex sample matrices and under more difficult environmental conditions are desirable to estimate the use of devices for molecular point-of-care detection of MRSA for military medical purposes.

\section{Funding Sources}

There has been no source of funding.

\section{Author's Contribution}

H.F. is the only author.

\section{Conflicts of Interest}

The author declares that there is no conflict of interest regarding the publication of this article.

\section{References}

1. Atherton ME. Outbreak of methicillin resistant Staphylococcus aureus in a Royal Naval hospital. J R Nav Med Serv. 1986;72:135-40.

2. Murray CK, Yun HC, Griffith ME, Thompson B, Crouch HK, Monson LS, et al. Recovery of multidrug-resistant bacteria from combat personnel evacuated from Iraq and Afghanistan at a single military treatment facility. Mil Med. 2009;174:598-604.

3. Curry JA, Maguire JD, Fraser J, Tribble DR, Deiss RG, Bryan C, et al. Prevalence of Staphylococcus aureus Colonization and Risk Factors for Infection Among Military Personnel in a Shipboard Setting. Mil Med. 2016;181:524-9.

4. Pagac BB, Reiland RW, Bolesh DT, Swanson DL. Skin lesions in barracks: consider community-acquired methicillin-resistant Staphylococcus aureus infection instead of spider bites. Mil Med. 2006;171:830-2.

5. Ellis MW, Hospenthal DR, Dooley DP, Gray PJ, Murray CK. Natural history of community-acquired methicillin-resistant Staphylococcus aureus colonization and infection in soldiers. Clin Infect Dis. 2004;39:971-9.

6. Monecke S, Slickers P, Ellington MJ, Kearns AM, Ehricht R. High diversity of Panton-Valentine leukocidin-positive, methicillin-susceptible isolates of Staphylococcus aureus and implications for the evolution of community-associated methicillin-resistant $S$. aureus. Clin Microbiol Infect. 2007;13:1157-64.

7. Tong A, Tong SY, Zhang Y, Lamlertthon S, Sharma-Kuinkel BK, Rude T, et al. Panton-Valentine leukocidin is not the primary determinant of outcome for Staphylococcus aureus skin infections: evaluation from the CANVAS studies. PLoS One. 2012;7:e37212

8. Berube BJ, Bubeck Wardenburg J. Staphylococcus aureus $\alpha$-toxin: nearly a century of intrigue. Toxins (Basel). 2013;5:1140-66.

9. Otto M. Staphylococcus aureus toxins. Curr Opin Microbiol. 2014;17:32-7.

10. Spaan AN, van Strijp JAG, Torres VJ. Leukocidins: staphylococcal bicomponent pore-forming toxins find their receptors. Nat Rev Microbiol. 2017; 15:435-47.

11. Murray CK, Roop SA, Hospenthal DR, Dooley DP, Wenner K, Hammock J, et al. Bacteriology of war wounds at the time of injury. Mil Med. 2006;171:826-9.

12. Leamer NK, Clemmons NS, Jordan NN, Pacha LA. Update: Communityacquired methicillin-resistant Staphylococcus aureus skin and soft tissue infection surveillance among active duty military personnel at Fort Benning GA, 2008-2010. Mil Med. 2013;178:914-20.

13. Ellis MW, Griffith ME, Dooley DP, McLean JC, Jorgensen JH, Patterson JE, et al. Targeted intranasal mupirocin to prevent colonization and infection by community-associated methicillin-resistant Staphylococcus aureus strains in soldiers: a cluster randomized controlled trial. Antimicrob Agents Chemother. 2007;51:3591-8.

14. Baum SE, Morris JT, Dooley DP, Watson R. Methicillin-resistant Staphylococcus aureus in an adult military beneficiary population lacking risk factors: susceptibility to orally available agents. Mil Med. 2003;168:126-30.

15. Page A, O'Rourke S, Brennan M, Clooney L, Le Blanc D, Griffin J, et al. Impact of Xpert MRSA/SA blood culture PCR assay on management of positive blood cultures in obstetric patients: a retrospective audit. Ir J Med Sci. 2017; 186:995-8.

16. Nielsen XC, Madsen TV, Engberg J. Evaluation of Xpert MRSA Gen 3 and BD MAX MRSA XT for meticillin-resistant Staphylococcus aureus screening in a routine diagnostic setting in a low-prevalence area. J Med Microbiol. 2017;66:90-5.

17. Rossney AS, Herra CM, Brennan GI, Morgan PM, O'Connell B. Evaluation of the Xpert methicillin-resistant Staphylococcus aureus (MRSA) assay using the GeneXpert real-time PCR platform for rapid detection of MRSA from screening specimens. J Clin Microbiol. 2008;46:3285-90.

18. Shaw AG, Vento TJ, Mende K, Kreft RE, Ehrlich GD, Wenke JC, et al. Detection of methicillin-resistant and methicillin-susceptible Staphylococcus aureus colonization of healthy military personnel by traditional culture, PCR, and mass spectrometry. Scand J Infect Dis. 2013;45:752-9.

19. Belmekki M, Mammeri H, Hamdad F, Rousseau F, Canarelli B, Biendo M. Comparison of Xpert MRSA/SA Nasal and MRSA/SA ELITe MGB assays for detection of the mecA gene with susceptibility testing methods for determination of methicillin resistance in Staphylococcus aureus isolates. J Clin Microbiol. 2013;51:3183-91.

20. Lourtet-Hascoëtt J, Bicart-See A, Félicé MP, Giordano G, Bonnet E. Is Xpert MRSA/SA SSTI real-time PCR a reliable tool for fast detection of methicillin-resistant coagulase-negative staphylococci in periprosthetic joint infections? Diagn Microbiol Infect Dis. 2015;83:59-62.

21. Buchan BW, Allen S, Burnham CA, McElvania TeKippe E, Davis T, Levi M, et al. Comparison of the next-generation Xpert MRSA/SA BC assay and the GeneOhm StaphSR assay to routine culture for identification of Staphylococcus aureus and methicillin-resistant $S$. aureus in positive-bloodculture broths. J Clin Microbiol. 2015;53:804-909.

22. Kelley PG, Grabsch EA, Farrell J, Xie S, Montgomery J, Mayall B, et al. Evaluation of the Xpert ${ }^{\mathrm{TM}}$ MRSA/SA Blood Culture assay for the detection of Staphylococcus aureus including strains with reduced vancomycin susceptibility from blood culture specimens. Diagn Microbiol Infect Dis. 2011;70:404-7.

23. Scanvic A, Courdavault L, Sollet JP, Le Turdu F. Interest of real-time PCR Xpert MRSA/SA on GeneXpert $\left({ }^{\circledR}\right)$ DX System in the investigation of staphylococcal bacteremia. Pathol Biol (Paris). 2011;59:67-72.

24. Spencer DH, Sellenriek P, Burnham CA. Validation and implementation of the GeneXpert MRSA/SA blood culture assay in a pediatric setting. Am J Clin Pathol. 2011;136:690-4. 
25. Romero-Gómez MP, Muñoz-Velez M, Gómez-Gil R, Mingorance J. Evaluation of combined use of MALDI-TOF and Xpert $\left({ }^{(B)}\right)$ MRSA/SA BC assay for the direct detection of methicillin resistance in Staphylococcus aureus from positive blood culture bottles. J Infect. 2013;67:91-2.

26. Henares D, Brotons P, Buyse X, Latorre I, de Paz HD, Muñoz-Almagro C. Evaluation of the eazyplex MRSA assay for the rapid detection of Staphylococcus aureus in pleural and synovial fluid. Int J Infect Dis. 2017;59:65-8.
27. Salimnia H, Fairfax MR, Lephart PR, Schreckenberger P, DesJarlais SM, Johnson JK, et al. Evaluation of the FilmArray Blood Culture Identification Panel: Results of a Multicenter Controlled Trial. J Clin Microbiol 2016;54:687-98.

28. Fhooblall M, Nkwanyana F, Mlisana KP. Evaluation of the BioFire ${ }^{\circledR}$ FilmArray ${ }^{\circledR}$ Blood Culture Identification Panel on positive blood cultures in a regional hospital laboratory in KwaZulu-Natal. Afr J Lab Med. 2016;5:411. 